\title{
In-Situ Lift-Out FIB Specimen Preparation for TEM of Magnetic Materials
}

\author{
B.W. Kempshall and L.A. Giannuzzi
}

Mechanical Materials and Aerospace Engineering, University of Central Florida, 4000 Central Florida Blvd, Orlando, FL 32816-2450

The focused ion beam (FIB) (ex-situ) lift-out technique has been used to prepare specimens for transmission electron microscopy (TEM) from numerous materials applications [1]. In this report, we use the in-situ lift-out technique [2] to prepare specimens from magnetic materials for TEM analysis. An FEI 200TEM FIB workstation equipped with an Omniprobe in-situ W probe was used for the specimen preparation. A Philips/FEI Tecnai F30 operating at $300 \mathrm{keV}$ was used for the TEM analyses.

FIG. 1 shows a cross-section bright field (BF) TEM micrograph of a $\mathrm{Nd}_{2} \mathrm{Fe}_{14} \mathrm{~B}_{1}$ sample containing $\mathrm{Nd}$, Dy, and Co. The sample was heated at $600^{\circ} \mathrm{C}$ for $30 \mathrm{~min}$. Many of the grains are elongated and consist of dimensions that are 50-100 nm in width and 100-300 nm in length. Preliminary results indicate that the smaller equiaxed grains that are on the order of $\sim 20 \mathrm{~nm}$ in dimension may consist of the $\alpha$ Fe phase. FIG. 2 shows a lattice image from a region in FIG. 1 which indicates that the FIB/TEM technique for these materials are suitable for further analysis.

FIG. 3 shows a BF TEM image from a single particle from a $\operatorname{Sm}\left(\mathrm{Co}_{7.05-\mathrm{x}} \mathrm{Cu}_{\mathrm{x}} \mathrm{Ti}_{0.25}\right), \mathrm{x}=0.8$ powder sample. We have previously shown that individual particles may be prepared directly by FIB lift-out techniques [3]. The specimen in FIG. 3 consisted of particles embedded in an epoxy matrix. Note that the single particle shown in FIG. 3 is polycrystalline in nature. A convergent beam electron diffraction pattern from the dark grain diffracting in the Laue condition shown in FIG. 3 is shown in FIG. 4. This CBED pattern most closely resembles a [0001] pattern that would be expected from either of the hexagonal structures (e.g., $\left.\mathrm{Sm}_{2} \mathrm{Co}_{17}, \mathrm{SmCo}_{5}\right)$ that should exist in this system.

High temperature superconducting $\mathrm{Bi}_{2} \mathrm{Sr}_{2} \mathrm{Ca}_{2} \mathrm{Cu}_{3} \mathrm{O}_{10}$ (BSCCO) was obtained from Colorado Superconductor, Inc. FIG. 5 shows a BF TEM micrograph from the BSCCO sample. The polycrystalline nature of the specimen in FIG. 5 is evident by the selected area diffraction pattern of the orthorhombic structure [4] shown in FIG. 6. These images show that FIB specimen preparation techniques are viable for TEM studies of magnetic materials. TEM studies may now be performed to elucidate the microstructural details of these materials [5].

References

[1] L.A. Giannuzzi, et al., Mat. Res. Soc. Symp. Proc., 480 (1997) 19.

[2] T. Kamino et al., Microsc. Microanal., 6 (Suppl. 2 ) (2000) 510.

[3] J. K. Lomness et al., Microscopy and Microanalysis, 7 (2001) 418.

[4] D.R. Mishra et al., Pramana J. Physics, 542 (2000) 317.

[5] The support of NSF DMR \#9703281 and Omniprobe is gratefully appreciated. Some samples were prepared by W. Liu and provided courtesy of Y. Liu. 


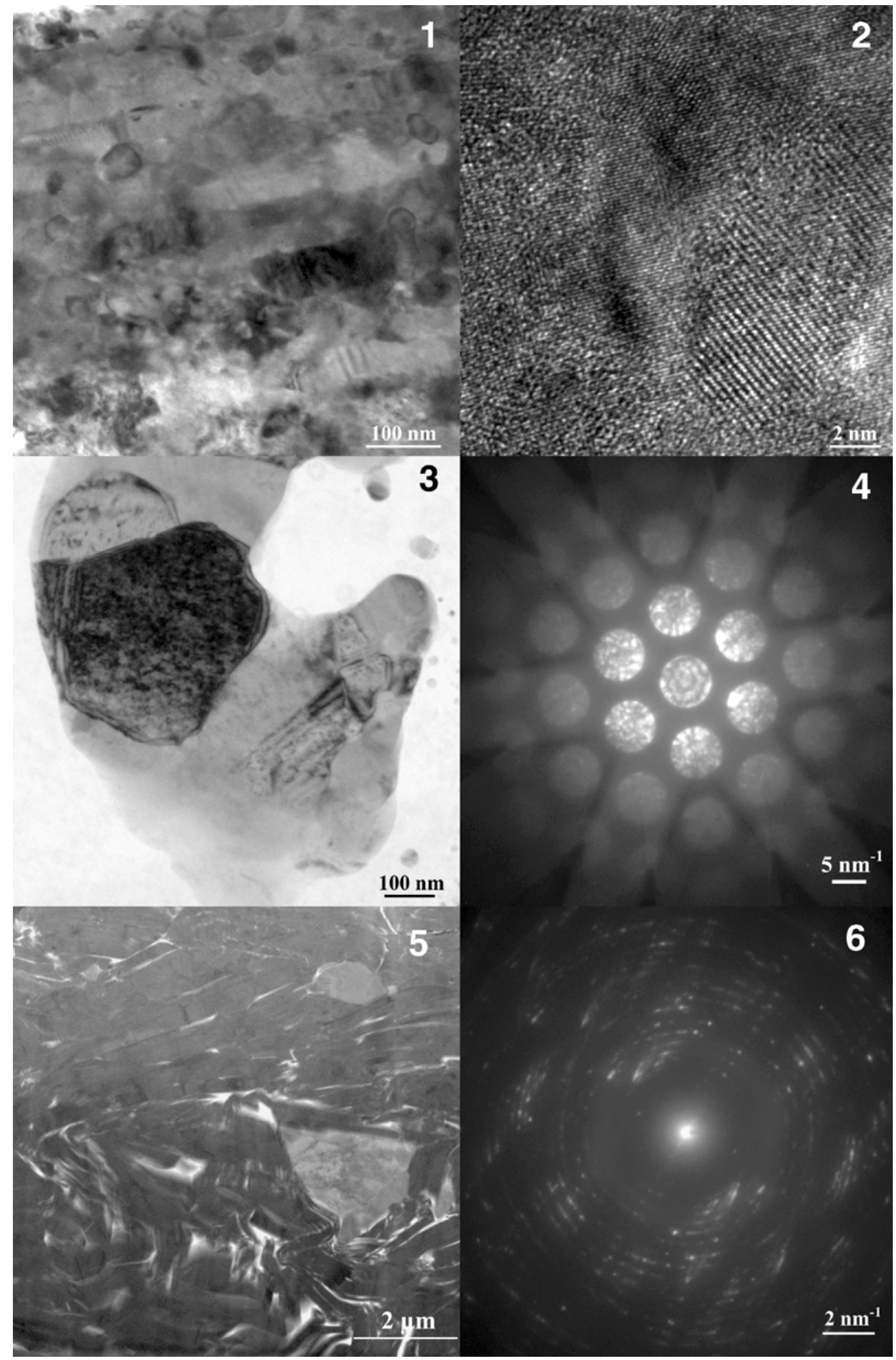

FIG. 1,2. BF TEM image and HREM image of an FeNdB thin film.

FIG. 3,4. BF TEM image and [111] CBED from a NDFCNB powder sample.

FIG. 5,6. BF TEM image and SADP of a BSCCO sample. 